\title{
Desarrollo de las capacidades coordinativas en niños: efectos de entrenamiento en el patinaje Development of coordination skills in children: effects of rollerblading training
}

Bertulfo Herrera Quiceno, Wilder Geovanny Valencia Sánchez, Diego Armando García Gómez, José Albeiro Echeverri Ramos

Universidad de Antioquia (Colombia)

Resumen. El objetivo del estudio fue determinar el efecto de un programa de aprendizaje de las capacidades coordinativas sobre el tiempo en la prueba de patinaje de 300 metros contra reloj individual y la coordinación general. Participaron 14 niños y niñas entre los seis y ocho años de edad que fueron asignados aleatoriamente al grupo experimental $(\mathrm{n}=7)$ o grupo control $(\mathrm{n}=7)$; la media de la edad del grupo experimental fue 8,1 años $(\mathrm{DE}=0.5)$ y la media del grupo control 7,6 años $(\mathrm{DE}=0.82)$; con una experiencia deportiva igual para ambos grupos (16 meses). El grupo experimental tuvo un entrenamiento adicional de coordinación antes de la sesión cotidiana durante 20 sesiones distibuidas en siete semanas. El programa constó de 15 ejercicios en escalerilla realizados en cada sesión asi: tres ejercicios con dos series de 10 repeticiones y recuperación de 30 segundos en la primera serie y dos minutos después de la segunda. El grupo control no recibió estímulo adicional y continuó con el entrenamiento cotidiano del patinaje. Se realizó pre y post-test donde se midió la velocidad con la prueba de $300 \mathrm{~m}$ contra reloj individual en patines y la coordinación de los niños con el test Korperkoordination Test fur Kinder. Los resultados mostraron que existen diferencias significativas en las comparaciones intra-grupo, sin embargo, al realizar la comparación inter-grupos, se constató que no existen diferencias significativas $(p=0.180)$ en velocidad, pero en la práctica del patinaje estas diferencias son relevantes porque determinan una disminución en el tiempo de una prueba contra reloj, que puede significar un cambió ecológico significativo.

Palabras claves: Coordinación, deporte, entrenamiento, patinaje, prueba de velocidad.

\begin{abstract}
The research objective was to determine the effect of a learning program for coordination skills on the time of the 300-meter rollerblading test against individual clock and on general coordination. A sample of 14 boys and girls between six and eight years old, who were randomly assigned to experimental group $(n=7)$ or control group $(n=7)$ participated in the study; average age of the experimental group was 8,1 years old $(\mathrm{SD}=0,5)$, whereas in the control group it was 7,6 years old $(\mathrm{SD}=0.82)$; participants from both groups had similar sports experience (16 months). During 20 sessions over seven weeks, the experimental group received additional coordination training before each daily session. The program consisted of 15 ladder exercises, where in each session three exercises were performed with two sets of 10 repetitions and recovery of 30 seconds in the first series and two minutes after the second. The control group did not receive additional stimulus and continued with daily rollerblading training. Pre- and post-test were performed, with speed being measured during the $300 \mathrm{~m}$ test against individual clock, whilst children's coordination was assessed with the Korperkoordination Test fur Kinder Test. The results showed significant differences in intra-group comparisons; however, when performing the inter-group comparison, no significant differences $(p=0.180)$ were found in speed. Nonetheless, in rollerblading practice these differences are relevant, because they determine a decrease in the time of a test against the clock, which may mean a significant ecological change.
\end{abstract}

Key words: Coordination, sport, training, skating, speed test.

\section{Introducción}

La práctica del patinaje requiere la adquisición, perfeccionamiento de habilidades y conceptos fundamentales que permitan al practicante dominar los elementos básicos de cada modalidad (Balyi \& Hamilton, 2004). Por tanto, su entrenamiento requiere de una periodicidad y progresión en las diferentes habilidades, con el fin de obtener un mejoramiento en el desempeño deportivo (Bompa, 2006).

Existen diferentes propuestas de entrenamiento a largo plazo (Echeverri, 2015) que apuntan al desarrollo del talento deportivo, entendido como un proceso que implica la interacción de varios factores. Algunas de estas propuestas buscan la permanencia del deportista en programas cuya duración asegure el desarrollo del potencial de rendimiento, teniendo en cuenta las edades y etapas del desarrollo motor.

Dentro de las propuestas de desarrollo deportivo a largo plazo (Balyi, 2001; Bruner, Erickson, Wilson \& Côté, 2010; Côté, 1999; Lloyd \& Oliver, 2012) existen coincidencias asociadas a la formación deportiva de acuerdo a las necesidades motrices (habilidades básicas, específicas y coordinativas)

Fecha recepción: 20-09-19. Fecha de aceptación: 27-01-20

Wilder Geovanny Valencia Sánchez

wilder.valencia@udea.edu.co relacionadas con la edad biológica de los participantes y disfrute del deporte. En el caso específico de la enseñanza y entrenamiento del deporte en las etapas de fundamentación deportiva, se busca que los aprendices disfruten y se apropien de las habilidades del deporte y el desarrollo eficiente de las capacidades coordinativas (Speedskating, 2016).

La coordinación en general es una capacidad indispensable para la vida de todo ser humano y determina de manera significativa la efectividad de sus acciones y comportamientos motrices; estas dependen de la armonización de todas las fuerzas externas e internas corporales, teniendo en cuenta todos los ejes de movimiento del aparato motor, para resolver adecuadamente una tarea propuesta (Camargo \& Yezid, 2014; De Boer, et al., 1987; Hands, Licari \& Piek, 2015; Hondt, et al., 2013; López, 2014; Petrone, 2006; Sahan, Erman \& Aðaoðlu, 2013).

De acuerdo a Vecino (2011) la coordinación es la facultad que tiene un individuo de ejecutar movimientos de la manera más precisa y ajustada posible al modelo o programa, combinando dos o más movimientos o segmentos corporales, ejecución que puede ser producto de la información recibida previamente y que depende del nivel de entrenamiento.

De esta manera, la coordinación influye en el aprendizaje de la técnica, la cual depende en gran medida de la habilidad que tiene el individuo de manejar su cuerpo con naturalidad 
y con movimientos coordinados (Da Silva, 2003). Es así que cualquier acción motriz como saltar, caminar, lanzar o atrapar, entre otras, necesitan de la coordinación para que los movimientos sean eficaces (Villa, 2011). En la edad escolar los estudiantes demuestran un mejor control y regulación de los movimientos en actividades cotidianas, cuando han recibido estímulos de entrenamiento coordinativo (Rosero, Palma \& Dávila, 2012).

En deportes que requieren el movimiento de todo el cuerpo, que demandan a su vez de una sincronía parcial o total, producto de la regulación de la actividad muscular (Petrone, 2006), la coordinación es uno de los factores de desarrollo fundamental para perfeccionar este tipo de habilidades (Garbolewski \& Starosta, 2013; Walaszek \& Nosal, 2014). La coordinación ha sido motivo de estudio a través del tiempo para determinar su influencia o relación con el aprendizaje de la técnica deportiva (Bustamante, 2007; Coral, 2011; Da Silva, 2003; de Souza, et al., 2015; Lopes, Maia, Silva, Seabra \& Morais, 2003; Mori, 2008; Ruiz, 2004; Soto, Cegarra, Cuartero, López \& Cantó, 2013; Turvey, 1990; Van Waelvelde, De Weerdt, De Cock \& Smits-Engelsman, 2004; Villa, 2011) y el éxito deportivo (Antón, 2009).

La práctica del patinaje exige el desarrollo de las habilidades motrices básicas, específicas y coordinativas, en la medida que se requiere el manejo de un elemento externo (patines), con una complejidad relacionada con la combinación de las habilidades antes señaladas (Lopes, et al., 2003). Éstas habilidades son susceptibles de ser mejoradas por medio de la práctica constante (Rosero, et al., 2012). Además, su desarrollo esta relacionado con el desempeño de las tareas propias del deporte (Soto, et al., 2013).

En el aprendizaje y entrenamiento del patinaje, las capacidades coordinativas juegan un papel muy importante en el desempeño del deportista (Caiza, Ortega \& Luis, 2013; Rosero, et al., 2012). Estas se deben aprender desde movimientos básicosque son necesarios para ejecuciones posteriores complejas (Hernando, 2014), que serán transferidas a los movimientos propios del patinaje. El desarrollo de planes o herramientas complementarias para mejorar las capacidades coordinativas se hacen indispensables en los procesos de entrenamiento en este deporte.

Las investigaciones muestran la influencia del entrenamiento de las capacidades coordinativas en edades escolares sobre el reconocimiento de la competencia motriz (García, et al., 2013; Lopes, et al., 2003), sobre la agilidad y la coordinación (Rosero, et al., 2012), los cambios en la coordinación de brazos y piernas con el aumento gradual de velocidad (Sparrow \& Newell, 1994), la influencia de la posición de inicio en carreras cortas de patinaje de velocidad con el orden de llegada (Maw, et al., 2006), la asociación entre los niveles de coordinación y la velocidad de reacción (Da Silva, 2003), la relación entre la capacidad de equilibrio y el rendimiento deportivo (Lopes, et al., 2010), la asociación entre la técnica y la velocidad (Hillis \& Holman, 2014) y el interés por el estudio de las capacidades coordinativas como medio para mejorar el desempeño deportivo de los niños (Cortéz, 2013; García, Pérez, Rodríguez \& Moral, 2013). De tal forma, que indagar por la influencia de programas con énfasis en la estimulación de las capacidades coordinativas como parte de la formación técnica de patinadores infantiles, representa una gran oportunidad y necesidad en el desarrollo deportivo a largo plazo en este deporte.

La indagación por medios empíricos (entrenamiento de las capacidades coordinativas sin patines) y su efecto en tiempo en pruebas de velocidad en patines representa una novedad en el entrenamiento en este deporte. Esto se debe, por la utilización de calzado (Elvira, et al., 2017) como los tenis deportivos en niños para realizar la intervención como posible influencia en el desempeño por el entrenamiento de las capacidades coordinativas.

Teniendo en cuenta, la fase inicial (seis a ocho años) del desarrollo del sistema nervioso y factores neurosensoriales de la coordinación, se considera éste el momento propicio para el mejoramiento de las capacidades coordinativas por medio de nuevas experiencias de movimiento (Muñoz, 2009; Balyi, 2001). Esta edad es la adecuada para una evaluación e inicio con los procesos de estimulación de las capacidades coordinativas (Quitério, et al., 2017) en procura de la mejora en la ejecución de los movimientos específicos del deporte.

El objetivo del presente estudio fue determinar el efecto de la implementación de un programa basado en capacidades coordinativas (grupo experimental, $n=7$ ) durante un período de siete semanas (20 sesiones) vs no recibirlo (grupo control, $n=7$ ), sobre el tiempo utilizado en la prueba de 300 metros contra reloj individual en 14 niños y niñas entre los seis y ocho años de edad.

\section{Método}

\section{Alcance del estudio}

Estudio explicativo que establece relaciones de causaefecto, entre la variable independiente (aprendizaje de la coordinación) y las variables dependientes (tiempo en la prueba de 300 m y coordinación).

\section{Diseño del estudio}

Estudio de tipo experimental con asignación aleatoria en paralelo (Friedman, Furberg \& DeMets, 2010). Los sujetos del grupo experimental fueron intervenidos mediante el programa de aprendizaje de la coordinación durante siete semanas (20 sesiones), mientras que el grupo control solo recibió el entrenamiento tradicional de patinaje. Ambos grupos fueron evaluados tanto al inicio como al final del tratamiento (pretest-postest).

\section{Participantes}

Participaron 14 niños y niñas entre seis y ocho años que practican patinaje en la liga de patinaje de Antioquia. Para calcular el tamaño de la muestra se adoptó la fórmula propuesta para la comparación de medias (Pita, 1996) para un delta (d) esperada de 0.6 segundos; donde la d esperada se obtuvo de una prueba con juveniles test-retest con un periodo de dos meses de entrenamiento entre pruebas; donde se obtuvo un d de 0.361 segundos. Se propuso 0.6 debido a la diferencia entre las poblaciones (Juveniles vs Infantiles sumado la experiencia), teniendo más posibilidad de cambio la población infantil debido a que su rendimiento es menor con $\mathrm{n}=7.53$

El cálculo de la potencia del estudio se hizo por medio de la comparación de medias bilateral a través de la fórmula 
propuesta por Pértegas \& Pita (2003) con una potencia del 95 $\%$ para un test bilateral con $n=7$. Para calcular el tamaño del efecto se centró en la diferencia estandarizada de medias o parámetro $d$ obtenida mediante la $g$ de Hedges ajustada ( $g$ ajust), siguiendo para ello las directrices de Ledesma, Macbeth, \& Cortada de Kohan (2008) y Tejero, Castro \& Balsalobre (2012). Debido a tres criterios favorables a la $g$ ajust: (1) estimación precisa e insesgada, (2) simplicidad de cálculo y (3) fácil interpretación del resultado. Se encontró $g=0.84$ que corresponde en la Tabla de la Función de Distribución de la Variable Normal Estándar a 0.8023, significa que el tamaño del efecto $80 \%$.

\section{Criterios de inclusión y exclusión}

Participaron patinadores entre seis y ocho años de edad con experiencia en el deporte de al menos un año, práctica regular de tres sesiones semanales, aval médico de participación; los padres firmaron el consentimiento informado y los participantes firmaron el asentimiento informado. Posteriormente, se sometieron a los criterios de exclusión, los cuales fueron: trastornos osteomusculares agudos, antecedentes de enfermedades como asma, problemas cardíacos, cointervenciones de deportes como gimnasia, porrismo, entre otros, participantes que usaran broncodilatadores $\mathrm{u}$ hormonas del crecimiento como insulina y niños con obesidad.

\section{Recolección de datos: Instrumentos}

En cada evaluación se midieron dos variables: velocidad y coordinación general. Para la medición del tiempo de cada individuo al recorrer $300 \mathrm{~m}$ contra reloj, se utilizó una foto celda con una exactitud de 0.001 milésimas de segundo. Se considera que para esa distancia los niños entre los seis y ocho años por sus condiciones de desarrollo físico; realizan esta actividad deportiva utilizando su sistema aeróbico (Balyi $\&$ Hamilton, 2004) que no representa un esfuerzo contraproducente para la muestra de esta investigación.

En el caso de la coordinación general se utilizó el test de coordinación motora para niños Korperkoordination Test fur Kinder (KTK) que, como lo indica Bustamante (2007), consiste en realizar una batería de ejercicios medida con una puntuación que depende de los aciertos o desaciertos que tenga el niño en cuatro pruebas: transposición lateral (TL), saltos monopedales (SM), saltos laterales (SL) y equilibrio a la retaguardia (ER). Fue creado por Kiphard \& Schilling (1974) quienes a partir de un conjunto de 150 tarea, encontrando que cuatro de ellas (TL, SM, SL, ER) se correlacionaban de forma positiva y elevada con las características de la coordinación motora. Este instrumento fue estandarizado en 1228 niños de ambos sexos entre los cinco y los 14 años. Se considera un instrumento confiable $(\mathrm{r}=0.60-0.99)$ (Iivonen, Sääkslahti \& Laukkanen, 2016) y es uno de los test más utilizados (Benjumea, et al., 2017).

\section{Control de los sesgos y aleatorización}

El personal encargado de la recolección de los datos fue capacitado con el fin de evitar sesgos de información; los equipos para la toma del tiempo estaban calibrados, ambos grupos tuvieron dos evaluaciones, una al inicio y otra al final del estudio. Todos los participantes fueron codificados por orden de llegada el primer día de evaluaciones garantizando la confidencialidad de los datos. Este orden fue tenido en cuenta para la asignación de los participantes a cada grupo. Los sujetos fueron asignados al azar por medio del Programa para Análisis Epidemiológico de Datos Tabulados (Epidat 3.1) para Windows 7, que suministró números aleatorios con una relación de 1:1, teniendo siete sujetos para el grupo experimental y siete sujetos para el grupo control, de esta manera los grupos quedaron balanceados. Los grupos tambien fueron codificados así: uno para el grupo experimental; dos para el grupo control. La asignación fue hecha por un investigador externo a los investigadores, quien no conocía los datos de los deportistas, solo se le proporciónó el listado codificado. Luego de realizar la asignación de los grupos se contactó vía telefónica a todos los sujetos y fueron notificados acerca del grupo al que fue asignado, enmascarando esta fase del estudio.

Se realizó un análisis por intención de tratamiento, donde los individuos fueron analizados en el grupo al que inicialmente fueron asignados en el estudio, independiente de la asistencia a la totalidad de las prácticas o si decidieron cambiar de grupo.

Las variables de confusión que se abordaron fueron: la edad que se tomó con la fecha de nacimiento, la talla se midió por medio de una balanza con estadiómetro marca Detecto con una precisión de $0.1 \mathrm{~cm}$, el peso se midió por medio de una balanza con estadiómetro marca Detecto cuya precisión es de $0.1 \mathrm{~kg}$, el estrato socio económico se midió por la última cuenta de los servicios del hogar, el Indice de Masa Corporal se midió con la relación de peso y talla; y la experiencia deportiva se contó en meses desde que el niño o niña empezó a patinar.

\section{Protocolo de intervención: Grupo experimental}

El protocolo tiene 15 ejercicios que fueron realizados durante 20 sesiones con una frecuencia aproximada de tres veces a la semana. La intensidad fue aumentando cada cinco sesiones de entrenamiento, donde cada ejercicio fue repetido cuatro veces durante el proceso. En cada sesión del programa de aprendizaje se realizaron tres ejercicios, donde cada ejercicio fue realizado dos series de 10 repeticiones; una repetición es el paso de la escalera coordinativa desde su inicio hasta el final, teniendo en cuenta que la escalera mide $5 \mathrm{~m}$ y que cada peldaño mide $45 \mathrm{~cm}$ x $45 \mathrm{~cm}$, después de cada serie de 10 repeticiones con un solo ejercicio el participante descansó 30 segundos, después de la primera serie con los tres ejercicios el participante descansó dos minutos y luego realizó la segunda serie (Tabla 1). Se consideró que la carga de trabajo apropiada teniendo evidencia empírica (Delignières, et al., 1998; Rosero, et al., 2012).

\begin{tabular}{|c|c|c|c|c|c|c|c|c|c|c|c|c|c|c|c|c|c|c|}
\hline \\
\hline $\begin{array}{l}\text { Pre- } \\
\text { test }\end{array}$ & 1 & \begin{tabular}{l|l}
2 & 3 \\
\end{tabular} & 4 & 5 & 6 & 7 & 8 & 9 & 10 & 11 & 12 & 13 & 14 & 15 & \begin{tabular}{l|l}
16 & 17
\end{tabular} & 18 & 19 & $20 \begin{array}{l}\begin{array}{l}\text { Pos- } \\
\text { test }\end{array} \\
\end{array}$ \\
\hline \multicolumn{19}{|c|}{ Estímulos y ejercicios } \\
\hline $\begin{array}{l}\text { Pre- } \\
\text { test }\end{array}$ & 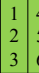 & & $\begin{array}{l}10 \\
11 \\
12\end{array}$ & $\begin{array}{l}13 \\
14 \\
15\end{array}$ & $\begin{array}{l}1 \\
2 \\
3\end{array}$ & $\begin{array}{l}4 \\
5 \\
6\end{array}$ & & \begin{tabular}{l|l}
10 \\
11 \\
12
\end{tabular} & \begin{tabular}{l|l}
13 \\
14 \\
15
\end{tabular} & \begin{tabular}{l|l}
1 \\
2 & \\
3 &
\end{tabular} & \begin{tabular}{l|l}
4 \\
5 \\
6
\end{tabular} & \begin{tabular}{l|l}
7 \\
8 \\
9
\end{tabular} & $\begin{array}{l}10 \\
11 \\
12\end{array}$ & \begin{tabular}{l|}
13 \\
14 \\
15
\end{tabular} & $\begin{array}{l}4 \\
5 \\
6\end{array}$ & $\begin{array}{l}7 \\
8 \\
9\end{array}$ & \begin{tabular}{l|l}
10 & 1 \\
11 & 1 \\
12 & 1
\end{tabular} & \begin{tabular}{l|l}
13 & Pos- \\
14 & Post \\
15 & test
\end{tabular} \\
\hline \multicolumn{19}{|c|}{$\begin{array}{l}\text { Cada ejercicio es representado por un número. Cada uno de los ejercicios se realizó dos series de } \\
10 \text { repeticiones para un total de } 60 \text { ejecuciones en cada sesión de clase. Cada cinco sesiones se } \\
\text { realizan los mismos ejercicios con aumento gradual de intensidad. }\end{array}$} \\
\hline \multicolumn{19}{|c|}{$\begin{array}{l}\text { Color verde: Aprendizaje de los ejercicios; color amarillo: Realiza los ejercicios a baja velocidad } \\
\text { naranja: Realiza los ejercicios a mediana velocidad; Rojo: Realiza los ejercicios a máxima } \\
\text { velocidad. }\end{array}$} \\
\hline
\end{tabular}


El grupo experimental, además de realizar su entrenamiento cotidiano (calentamiento de 30 minutos, una parte principal orientada hacia el mejoramiento de la técnica del patinaje en recta y en curva, con una duración de una hora y 30 minutos y la parte final de 30 minutos) fue sometido a un programa de aprendizaje adicional antes del entrenamiento cotidiano de aproximadamente 15 minutos centrado en las capacidades coordinativas. Los estimulos realizados por este grupo se pueden observar en estricto orden en la Figura 1.

El grupo control realizó el entrenamiento habitual descrito anteriormente al mismo tiempo que el grupo experimental.

\section{Aspectos éticos}

Los riesgos del estudio fueron definidos como mínimos y propios de la práctica deportiva según la Resolución 008430 de 1993, artículo 11 numeral b del Ministerio de Salud de la República de Colombia (1993) y la Declaración de Helsinki actualizada en fortaleza en el 2013 (Declaración de Helsinki de la AMM - Principios éticos para las investigaciones médicas en seres humanos., 2013). Se garantizó el respeto a la autonomía, la dignidad y la intimidad de los participantes que ingresaron de manera voluntaria y se podían retirar en cualquier momento. Este estudio se realizó con aval de participación a través del consentimiento informado del representante legal y asentimiento para los menores. El proyecto de investigación fue avalado por el comité de ética del Instituto de Educación Física de la Universidad de Antioquia, resolución 113 del consejo del Instituto del 01 de junio de 2015.

\section{Análisis estadístico}

En el análisis univariado a las variables cuantitativas se aplicó la prueba de normalidad de Shapiro-Wilk ( $\leq 30)$; los datos con distribución normal, se resumieron con medias y desviaciones estándar; las variables con distribución no normal, se resumieron con medianas y rangos intercuartílicos, además las variables cualitativas se resumieron con proporciones. Se realizó un análisis intragrupo, dependiendo de la distribución de los datos para medidas repetidas sea $t$ de Student o la prueba no paramétrica de rangos con signos de Wilcoxon.

En el análisis bivariado se comparó el resultado en el tiempo del grupo experimental y grupo control. A los datos con distribución normal se aplicó una prueba paramétrica $t$ de Student para variables independientes;
Desplazamiento lateral: Este ejercicio se realiza en una escalera coordinativa de izquierda a derecha pasando los pies en el orden indicado, iniciando con el derecho, luego se realiza en sentido contrario iniciando con el pie izquierdo.

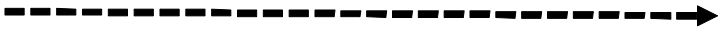

818989898989

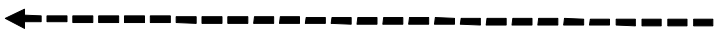

Desplazamiento frontal: Este ejercicio se realiza en una escalera coordinativa de frente pasando los pies en el orden indicado, iniciando con el derecho, luego se realiza de la misma manera pero iniciando cada secuencia con el pie izquierdo.

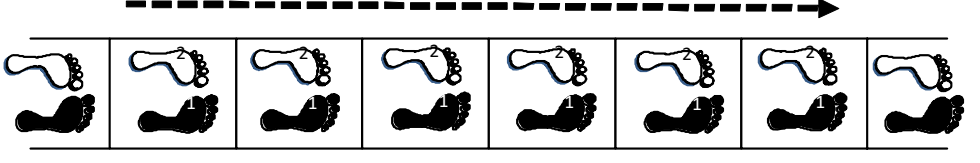

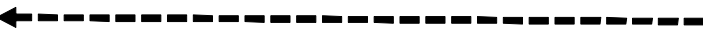

Desplazamiento frontal a tres apoyos: Este ejercicio se realiza en una escalera coordinativa de frente pasando los pies en el orden indicado iniciando con cualquiera de los dos pies, teniendo en cuenta que en cada nuevo cuadro debe iniciar con el pie contrario.

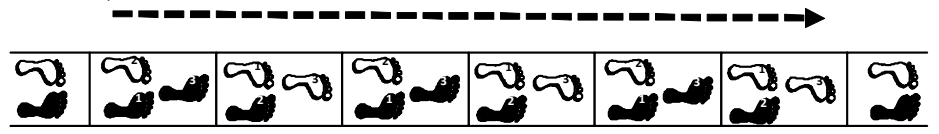

Saltos laterales con apoyo uni-podal: Este ejercicio se realiza con 12 aros separados a 20 centímetros entre uno y otro en la misma hilera y a 40 centímetros entre las dos hileras medidos desde el borde del aro, no importa con cual pie inicie, ya que el ejercicio es alternado, claro está que es dependiendo del aro que este más cerca del deportista, al realizar el ejercicio se debe pasar todo el cuerpo hasta donde se ubica el pie que está en el piso.

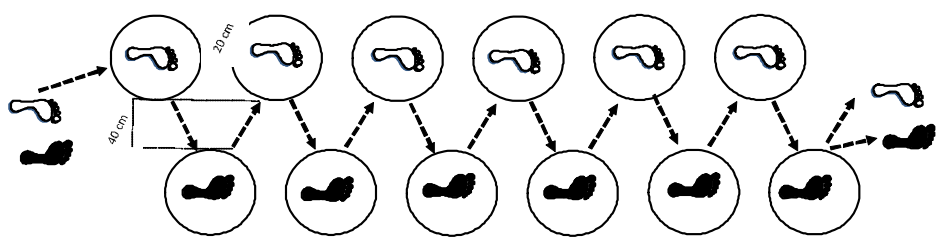

Saltos laterales con cruce de las piernas: Este ejercicio se realiza con 12 aros ubicados separados a 20 centímetros entre uno y otro de la misma hilera y a 40 centímetros entre las dos hileras medidos desde el borde del aro, no importa con cual pie inicie, ya que el ejercicio es alternado, claro está que es dependiendo del aro que esté más cerca del deportista, al realizar el ejercicio se debe pasar todo el cuerpo hasta donde se ubica el pie que está en el piso.

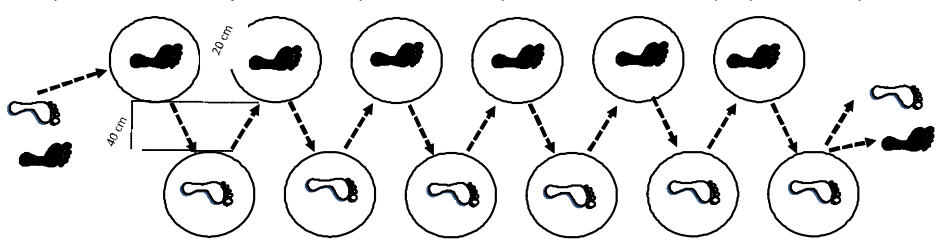

Desplazamiento lateral a tres apoyos: Este ejercicio se realiza en una escalera coordinativa de frente con desplazamientos laterales, pasando los pies en el orden indicado iniciando con cualquiera de los dos o por cualquier lado, teniendo en cuenta que en cada nuevo cuadro debe iniciar con el pie contrario, entrando y saliendo de los cuadros de manera lateral.

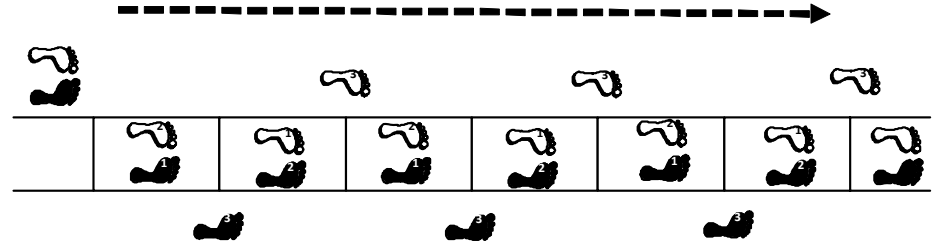

Desplazamiento frontal a dos pies: Este ejercicio se realiza en una escalera coordinativa de frente con un desplazamiento a dos pies, en el orden indicado procurando no tocar las líneas de la escalera.
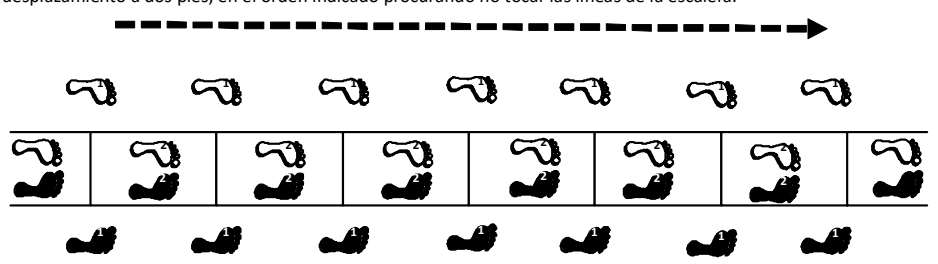

Desplazamiento lateral con apoyo de ambos pies tanto adentro como afuera: Este ejercicio se realiza en una escalera coordinativa de frente con desplazamientos laterales con el apoyo de los pies alternados, pasando los pies en el orden indicado iniciando con cualquiera de los dos o por cualquier lado, teniendo en cuenta que en cada nuevo cuadro debe iniciar con el pie contrario, realizando las entradas y las salidas.

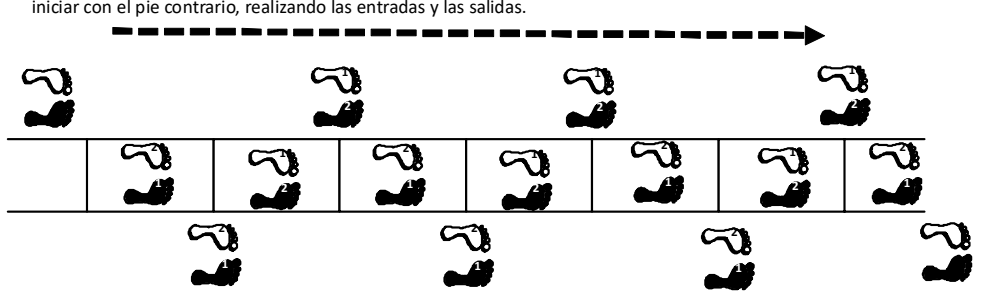


Desplazamiento lateral con apoyo de ambos pies tanto adentro como afuera pies juntos: Este ejercicio se realiza en una escalera coordinativa de frente con desplazamientos laterales con el apoyo de los pies alternados, pasando los pies en el orden indicado iniciando con cualquiera de los dos o por cualquier lado, teniendo en cuenta que en cada nuevo cuadro debe iniciar con el pie contrario, realizando las entradas y las salidas.

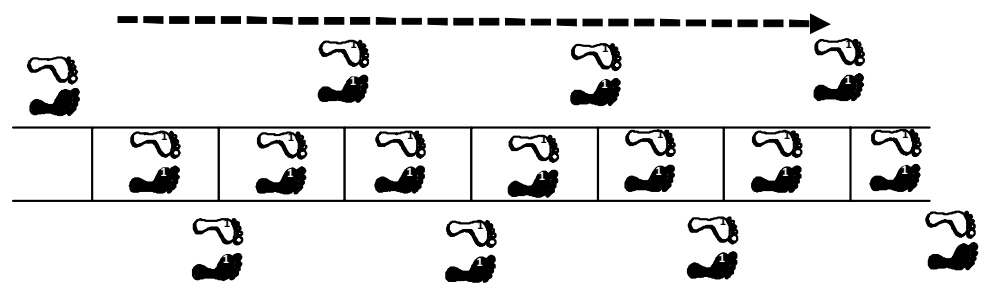

Cruzado por atrás: Este ejercicio se realiza en una escalera coordinativa de frente con desplazamientos frontales, apoyando en la mitad del cuadro siempre el mismo pie, y el pie contrario pasa de lado a lado por atrás de la otra pierna, así mismo se realiza con el otro pie.
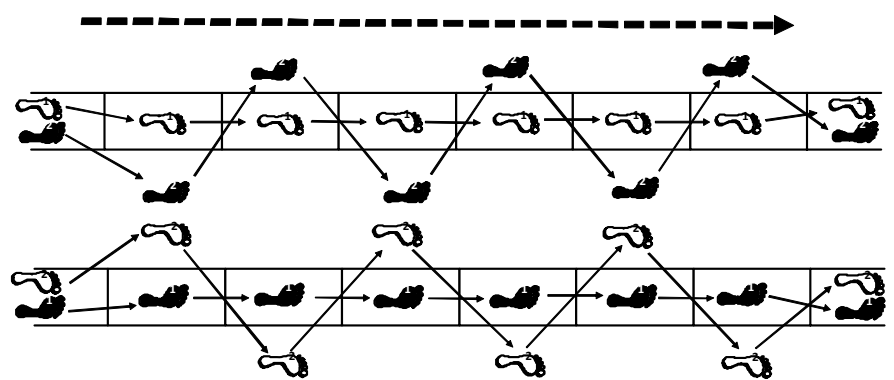

A un solo pie: Este ejercicio se realiza en una escalera coordinativa de frente con desplazamientos frontales, apoyando en la mitad del cuadro siempre el mismo pie, y el pie contrario se apoya afuera de la escalera, se realiza con un pie, luego con el otro, por el otro lado.

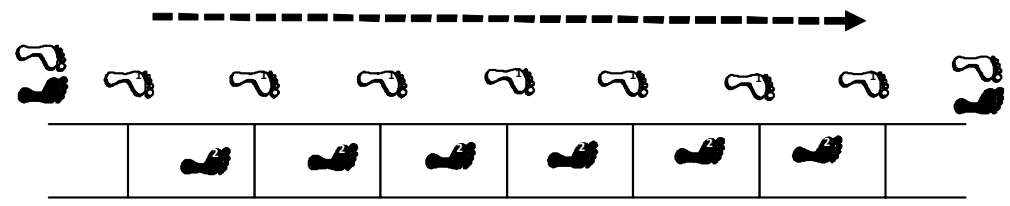

Combinación de movimientos con giro del cuerpo: Este ejercicio se realiza en una escalera coordinativa de frente con desplazamientos frontales y con giros del mismo cuerpo, cada vez que termine la secuencia debe cambiar de sentido, procurando no tocar las líneas de la escalerilla.

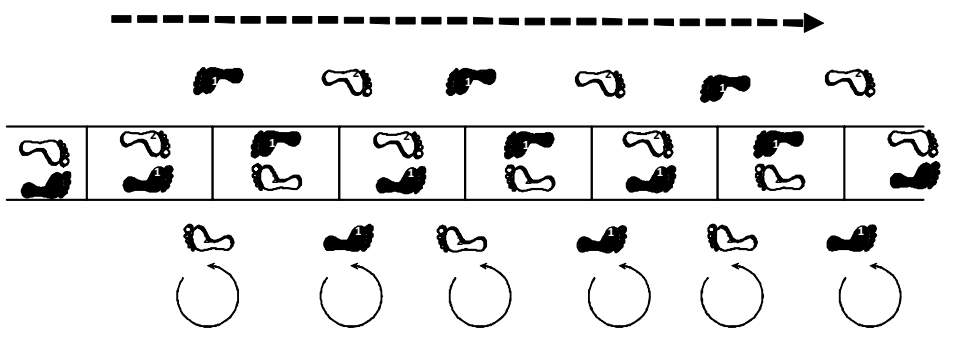

Desplazamiento lateral simultaneo En tijeras: Este ejercicio se realiza en una escalera coordinativa de frente con desplazamientos laterales, donde en movimiento de tijera los pies caen al piso al mismo tiempo pero siempre en un lugar diferente, puede iniciar con los pies en cualquier orden, cualquiera adelante.

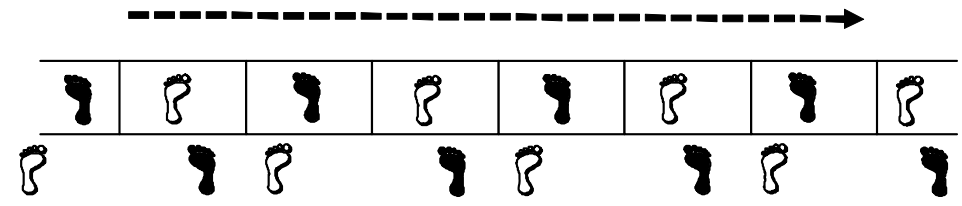

Desplazamiento frontal con medio giro cada cuadro de la escalera: Este ejercicio se realiza en una escalera coordinativa de frente con desplazamientos laterales y frontales combinados, donde el cuerpo debe estar en constante movimiento con un cabio en la rotación del tronco.

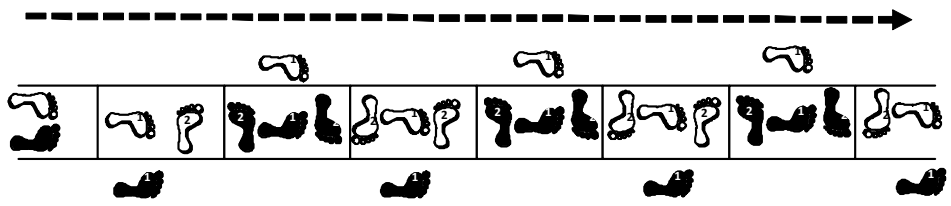

Desplazamiento frontal con saltos laterales a dos pies: Este ejercicio se realiza en una escalera coordinativa de frente con desplazamientos frontales y saltos laterales con ambos pies cayendo en distintos lugares, según la ilustración

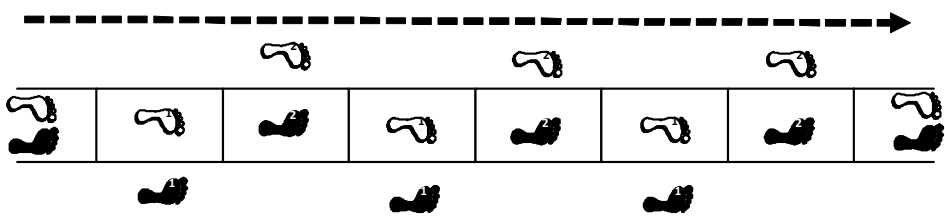

los datos con distribución no normal se compararon con una prueba no paramétrica U de Mann-Whitney. También se hizo una prueba de correlación entre las variables tiempo en la prueba de $300 \mathrm{~m}$ contra reloj y la prueba de coordinación para determinar la asociación. Se aplicó el coeficiente de correlación de Spearman. Los porcentajes de cambio fueron calculados así: ([valor del post test - valor del pre test]/ valor del pre test) $x$ 100. Se tuvo en cuenta una confiabilidad del $95 \%$ y una $p \leq 0.05$. Se utilizó el paquete estadístico IBM SPSS Versión 24.

\section{Resultados}

Los participantes del estudio $(n=14)$ fueron asignados aleatoriamente al grupo experimental o grupo control. La media de la edad del grupo experimental fue 8.1 años $(\mathrm{DE}=0.5)$ y la media del grupo control 7.6 años $(\mathrm{DE}=0.82)$. La experiencia deportiva fue igual para ambos grupos con medianas de 16 meses; el grupo control tuvo cinco niñas y dos niños, mientras que en el grupo experimental solo participaron niñas $(n=7)$. La variable dependiente fue similar para ambos grupos en el tiempo en la prueba (40.29 vs $41.45 \mathrm{~s}$ ).

Después de realizar los análisis estadísticos para comprobar las diferencias basales, con la prueba U de Mann-Whitney se encontró que no hubo diferencias estadísticamente significativas ( $\mathrm{p}>0.05)$, esto corresponde a que los grupos quedaron homogéneos. Así que la distribución al azar por medio del método aleatorio simple, funcionó cumpliendo así el objetivo de homogeneidad (Tabla 2).

\section{Análisis Bivariado}

El análisis intragrupo se realizó mediante la prueba de rangos de Wilcoxon por la distribución de los datos de ambos grupos. La mediana del grupo experimental en el pretest de la prueba $300 \mathrm{~m}$ Contra Reloj Individual (CRI) fue $40.29 \mathrm{~s}(\mathrm{RI}=9.30)$ y el postest fue $38.32 \mathrm{~s}(\mathrm{RI}=8.54)$, al aplicar el estadístico arrojó un valor $p=0.018(\mathrm{Z}=-$ 3.233) donde existen diferencias estadísticamente significativas. Mientras que la mediana del grupo control en el pretest de la prueba $300 \mathrm{~m}$ CRI fue $41,45 \mathrm{~s}$ ( $\mathrm{RI}=7.67)$ y el postest fue $40.89 \mathrm{~s}(\mathrm{RI}=7.77)$, al aplicar el estadístico arrojó un valor $p=$ $0.028(Z=-2.197)$ donde existen diferencias estadísticamente significativas (Tabla 3 ).

El análisis intragrupo para la variable de coordinación anlizado mediante pun- 
Tabla 2.

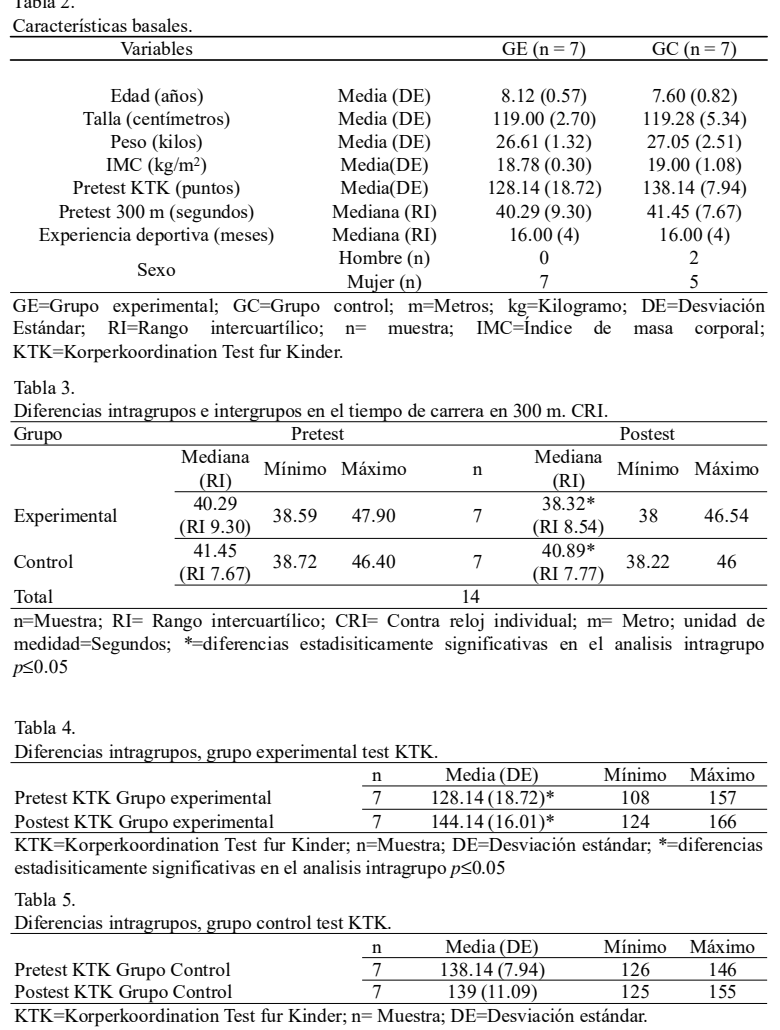

tos, se realizó con $t$ de Student para medidas repetidas dado que ésta tuvo una distribución normal. Para el grupo experimental el estadístico arrojó un valor $p=0.018$ que demuestra diferencias estadísticamente significativas, en la comparación intragrupo, la media del pretest fue de $128.14(\mathrm{DE}=18.72)$ y la media del postest fue de 144.14 (DE=16.01) (Tabla 4).

Por su parte el estadístico para el grupo control arrojó un valor $p=0.674$ mostrando que no existen diferencias estadísticamente significativas en la comparación intragrupo, la media del pretest fue de 138.14 ( $\mathrm{DE}=7.94)$ y la media del postest fue de 139 ( $\mathrm{DE}=11.09)$ (Tabla 5).

Para la comparación de ambos grupos en la prueba $300 \mathrm{~m}$ CRI se realizó un contraste de hipótesis mediante la prueba U de Mann-Whitney. La mediana del grupo experimental en el postest fue $38.32 \mathrm{~s}(\mathrm{RI}=8.54)$ mientras que la mediana del grupo control fue $40.89 \mathrm{~s}(\mathrm{RI}=7.78)$. Al realizar la comparación entre grupos la prueba de hipótesis dio un valor de $p=$ 0.18 en el cual no existen argumentos para rechazar la $\mathrm{H}_{\mathrm{o}}$ entonces desde el punto de vista estadístico no existen diferencias significativas entre el grupo control y el grupo experimental (Tabla 3). Sin embargo, desde el punto de vista práctico la diferencia que el grupo experimental obtuvo en la prueba $300 \mathrm{~m}$ después de haber implementado el programa de aprendizaje de las capacidades coordinativas es un cambio realmente ecológico significativo (Planelles, 2015) o en otras palabras, podría significar una medalla en competencia porque son más veloces.

Finalmente se realizó la prueba de correlación no paramétrica de Rho de Spearman entre las variables tiempo en la prueba $300 \mathrm{~m}$ CRI y puntaje de coordinación KTK del grupo experimental para establecer relaciones entre ambas variables. El valor de Rho de Spearman para el grupo experimental fue 0.571 ( $p=0.180$ ), que demuestra una relación posi- tiva moderada entre el tiempo en la carrera $300 \mathrm{~m}$ y la coordinación, es decir, cuanto mayor es el puntaje en la coordinación, menor es el tiempo de carrera en la prueba de $300 \mathrm{~m}$ con una magnitud moderada de asociación del $57 \%(\mathrm{Rho}=0.57)$ de la máxima posible; sin embargo, la asociación no llega a ser estadísticamente significativa $(p=0.180)$.

Por otro lado, el grupo control, obtuvo un coeficiente de correlación $-0.455(p=0.305)$ que demuestra una relación negativa moderada entre el tiempo en la carrera $300 \mathrm{~m}$ y la coordinación, es decir, cuanto menor es el puntaje en la coordinación, menor es la influencia que tiene en el tiempo de carrera de la prueba de $300 \mathrm{~m}$ con una magnitud moderada de asociación del $45 \%(\mathrm{Rho}=-0.455)$ de la máxima posible, $\sin$ embargo la asociación no llega a ser estadísticamente significativa $(p=0.305)$.

\section{Discusión}

La práctica repetitiva y la experiencia deportiva traen como resultado la mejora en el desempeño coordinativo (Delignières, et al., 1998; Sterkowics et al., 2012), teniendo su máximo desarrollo en edades tempranas (seis y ocho años) (Mateo \& Sáez, 2004) consideradas como la etapa de desarrollo intenso o critico de estas capacidades (Antón, 2009; Quitério, et al., 2017). La investigación sobre las capacidades coordinativas en el entrenamiento deportivo ha sido de gran interés donde diferentes autores han relacionado los niveles de coordinación con la ejecución técnica y su eficacia (Benjumea, et al., 2017; Da Silva, 2003; Stojanoviæ, et al., 2014), y los niveles de coordinación con el aprendizaje de la técnica y su perfeccionamiento (Garbolewski \& Starosta, 2013; Hillis \& Holman, 2014; Lyakh, et al., 2011; Villa, 2011; Walaszek \& Nosal, 2014).

La coordinación puede ser mejorada por medio de la práctica, ya sea de un deporte, varios o por medio de una actividad física cualquiera (Cvetkovic, et al., 2011; Hodges, Hayes, Horn \& Williams, 2005; Fransen, et al., 2012) y está estrechamente relacionada con el desarrollo de las habilidades motoras (Radwan, 2014).

Walaszek \& Nosal, (2014) han podido constatar cambios en la coordinación a partir de procesos de entrenamiento de hasta un año. De igual forma, Hodges, et al., (2005) evidenciaron mejoras de la coordinación en procesos cortos de entrenamiento de hasta nueve días en deportistas novatos.

Los cambios en los niveles de coordinación como resultado de la práctica deportiva se han podido constatar en un gran número de investigaciones (Cvetkovic, et al., 2011; Delignières, et al., 1998; Hodges, et al., 2005; Lopes, et al., 2003; Rosero, et al., 2012; Sterkowics, et al., 2012; Walaszek $\&$ Nosal, 2014). Sin embargo, son pocas las investigaciones que han indagado por la influencia del entrenamiento de la coordinación en la mejora de las habilidades deportivas y su repercusión en el desempeño deportivo específico, como la que proponen Montenegro \& Ramos (2015). En el caso de esta investigación, la mejora en la carrera de $300 \mathrm{~m}$ se ve reflejada en la disminución del tiempo utilizado para recorrer dicha distancia.

En el presente estudio se evaluó el rendimiento en tiempo, en una carrera corta de $300 \mathrm{~m}$ CRI en patines, después de aplicar un entrenamiento complementario de las capacida- 
des coordinativas a un grupo y se comparó con otro grupo que no recibió el estímulo adicional; las edades estaban comprendidas entre los seis y ocho años de edad con una experiencia deportiva de mínimo un año. Los resultados mostraron que el grupo sometido al programa de aprendizaje de las capacidades coordinativas, obtuvo un mejor desempeño en la carrera de $300 \mathrm{~m}$. Estos resultados pueden ser explicados por una mejora en la técnica de carrera (Da Silva,2003; Garbolewski \& Starosta, 2013; Stojanoviæ, et al., 2014; Villa 2011; Walaszek \& Nosal, 2014). Sin embargo, al realizar la comparación estadística intergrupos se pudo constatar que no existen diferencias significativas $(p=0.180)$. De igual forma, los resultados del estudio se pueden explicar por las posibles adaptaciones del Sistema Nervicoso Central (SNC) y una mejora de la coordinación muscular y la técnica, similar a los hallazgos de Hillis \& Holman, (2014) en su investigación con patinadores prepúberes.

Teniendo en cuenta que el patinaje de velocidad es un deporte donde la diferencia de resultados en pruebas de velocidad entre un corredor y otro es muy pequeña, se considera desde el punto de vista práctico de este deporte, una mejora del $6.2 \%$, (resultado del porcentaje de cambio) del grupo experimental sobre el grupo control es muy importante.

El resultado de la investigación soporta la hipótesis que el programa de aprendizaje de las capacidades coordinativas mejora el tiempo en una prueba de velocidad, resultado que puede estar asociado a la mejora en la técnica del deportista, (Cortéz, 2013; Lech, et al., 2014; Montenegro \& Ramos, 2015). Además, es posible un incremento en la velocidad de reacción asociado con el nivel de coordinación (Da Silva, 2003).

Así, este estudio realizó 20 sesiones que corresponden a 1200 repeticiones adicionales a la intervención cotidiana de los partinadores encontrando diferencias estadísticas en el análisis intragrupo, al igual que el estudio de Montenegro \& Ramos, (2015) con una población similar. Sin embargo, se recomienda ampliar el programa de coordinación, como en el estudio realizado por Walaszek \& Nosal, (2014) donde investigaron el nivel de coordinación motora en un grupo de 64 niños de ocho años, divididos en grupo experimental y control, donde tras un año de práctica y evaluados con el test de coordinación de Starosta, el grupo experimental mostró una mayor coordinación. En contraste Rosero, et al., (2012) encontró ventajas en la agilidad y la coordinación del grupo experimental sobre el control en tan solo ocho sesiones.

Cada uno de los grupos aumento el rendimiento al finalizar el proceso, lo cual puede ser explicado, por el entrenamiento específico de patinaje que recibieron durante el periodo de tiempo que duró la investigación (20 sesiones), en coherencia con el estudio de Lopes, Lopes, Santos \& Pereira, (2010) donde evaluaron la relación existente entre la actividad física, las habilidades motrices básicas y la coordinación motora, en un grupo de 21 niños con una edad promedio de 6.38 años $(\mathrm{DE}=0.50)$. Los resultados mostraron una relación directa con la actividad física, es decir, que a mayor actividad física realizada mayor fue la coordinación motora y también las habilidades motrices básicas. Así, por el solo hecho de entrenar incrementa la coordinación (Cvetkovic, et al., 2011; Hodges, Hayes, Horn \& Williams, 2005; Fransen, et al., 2012).

\section{Conclusiones}

El programa de aprendizaje coordinativo complementario en zapato deportivo, adicional a la práctica regular en patines del grupo experimental a partir de la comparación de los resultados pre y postest no mostró diferencias estadísticamente significativas, pero demostró tener influencia en la prueba de velocidad comparado con el grupo control. De igual forma, el grupo experimiental obtuvo una mejora significativa en la evaluación de la coordinación motora general con respecto al grupo control.

El aprendizaje de las capacidades coordinativas en el grupo experimental mejoró el rendimiento en patinaje de la prueba de velocidad $300 \mathrm{~m} \mathrm{CRI}(6.2 \%)$, debido posiblemente a la mejora en la ejecución técnica influenciada por el incremento de la coordinación.

A pesar que el estudio demostró que no hay diferencias estadísticamente significativas entre el grupo que realizó el programa de aprendizaje complementario de las capacidades coordinativas (grupo experimental) comparado con el grupo que no las realizó (grupo control), se puede concluir que la diferencia encontrada es favorable para la práctica, porque una diferencia de milésimas de segundo representa una ganancia importante en este tipo de deportes. Por lo tanto, el entrenamiento de velocidad para los patinadores antes de la pubertad debe centrarse en la adaptación del SNC a una variedad de movimientos, basado en las capacidades coordinativas, que amplifican ganancias de velocidad a través del entrenamiento específico de esta capacidad.

En próximos estudios es necesario ampliar el tamaño de la muestra, la cual fue una limitación en el presente estudio y análisis al momento de realizar comparaciones donde el tamaño de la muestra puede afectar los resultados (MartínezGonzález, et al., 2014).

\section{Agradecimientos}

Agradecimientos a la Universidad de Antioquia, Club de patinaje PAEN de Envigado y Liga Antioqueña de Patinaje por la infraestructura para desarollar el proyecto.

\section{Declaración de intereses}

Los autores declaran que no tiene ningún tipo de conflicto de intereses.

\section{Referencias}

Antón, G. J. L. (2009). El entrenamiento de las capacidades coordinativas en deportes de equipo: Bases de aplicación y tratamiento en la formación del jugador. Funámbulos editores.

Declaración de Helsinki de la AMM - Principios éticos para las investigaciones médicas en seres humanos., 9 (2013) (testimony of Asociación Médica Mundial).

Balyi, I. (2001). Sport system building and long-term athlete development in British Columbia. Coaches Report, 8(1), 2228.

Balyi, I., \& Hamilton,A. (2004). Long-term athlete development: Trainability in childhood and adolescence. Windows of 
opportunity. Optimal trainability. Victoria: National Coaching Institute British Columbia \& Advanced Training and Performance Ltd, 194.

Benjumea, J. M. C., Afonso, J. R., Pineda, S. M., \& FernándezTruan, J. C. (2017). Test de coordinación motriz 3JS: Cómo valorar y analizar su ejecución. Retos: nuevas tendencias en educación física, deporte y recreación, 32, 189-193.

Bompa, T. O. (2006). Periodización del entrenamiento deportivo. Editorial Paidotribo.

Bruner, M. W., Erickson, K., Wilson, B., \& Côté, J. (2010). An appraisal of athlete development models through citation network analysis. Psychology of Sport and Exercise, 11(2), 133-139.

Bustamante, V.A. (2007). Análisis interactivo de la coordinación motora, actividad física y del índice de masa corporal en escolares peruanos. [Tesis de Maestría]. Universidad de Porto.

Caiza, Ortega, \& Luis. (2013). Estudio de las capacidades coordinativas y su influencia en los fundamentos técnicos del fútbol en los niños y niñas de 10 - 12 años de las escuelas fiscales 9 de octubre y Túpac Yupanqui de la parroquia de san Antonio de Ibarra. [Tesis de pregrado]. Universidad Técnica del Norte.

Camargo, V., \& Yezid, B. (2014). Características morfo-funcionales y motoras en jugadores de balonmano categoría juvenil de la selección de Yumbo-Valle [Thesis]. http:// bibliotecadigital.univalle.edu.co/handle/10893/7104

Coral, T. D. F. (2011). Diseñary aplicar instrumentos para evaluar las Capacidades Coordinativas de los futbolistas profesionales. [Tesis de pregrado]. Universidad San Francisco de Quito.

Cortéz,A. O. (2013). Las capacidades coordinativas y su influencia en la ejecución de los lanzamientos del baloncesto en los deportistas de la categoría sub 14 de la liga deportiva cantonal de Mocha. [Tesis de Maestría]. Universidad Técnica de Ambato.

Côté, J. (1999). The influence of the family in the development of talent in sport. The sport psychologist, 13(4), 395-417.

Cvetkovic, M., Jaksic, D., Orlic, D., Obradovic, J., \& Pantovic, M. (2011). Effects of skating course on transformation of particular motor abilities. Short Scientific Paper, 2, 186-191.

Da Silva, M. M. E. (2003). Coordenação Motora e Velocidade de Reacção [Tesis de Maestría]. Universidad de Porto.

De Boer, R. W., Cabri, J., Vaes, W., Clarijs, J. P., Hollander,A. P., De Groot, G. \& Van Ingen, S. G. (1987). Moments of force, power, and muscle coordination in speed-skating. International journal of sports medicine, 8(6), 371-378.

De Souza, P. R. P., De Carvalho, S. G, Rodrigues, G. M., Cymrot, R., Saeta, B. R. P., \& Blascovi-Assis, S. M.(2015). Coordenação motora e ritmo circadiano em meninos com características variadas no desenvolvimento. Revista Neurociencia, 23(3), 342-348.

Delignières, D., Nourrit, D., Sioud, R., Leroyer, P., Zattara, M., \& Micaleff, J. P. (1998). Preferred coordination modes in the first steps of the learning of a complex gymnastics skill. Human Movement Science, 17, 221-241.

Echeverri, R. J. A. (2015). Modelos de desarrollo deportivo y factores condicionantes relacionados con el desarrollo del talento deportivo. http://viref.udea.edu.co, 4(2), 69-85.

Elvira, J. L. L., Plaza, D. L., Valenciano, A. L., \& Bueno, C. M. (2017). Influencia del calzado en el movimiento del pie durante la marcha y la carrera en niños y niñas de 6 y 7 años. Retos: nuevas tendencias en educación física, deporte y recreación, 31(1), 128-132.

Friedman, L. M., Furberg, C. D., \& DeMets, D. L. (2010). Fundamentals of Clinical Trials. Springer New York. http:// link.springer.com/10.1007/978-1-4419-1586-3

Garbolewski, K., \& Starosta, W. (2013). Level and conditions of global motor coordination and jumping abilities among polish and german national team water-polo players. Acta Kinesiologica, 2, 7-9.

García, C., Pérez, S. E., Rodríguez, J. J., \& Moral, J. E. (2013). Relación de las capacidades coordinativas con la competencia motriz autopercibida en adolescentes. Trances, 5(3), 213228.

Hands, B., Licari, M., \& Piek, J. (2015). A review of five tests to identify motor coordination difficulties in young adults. Research in Developmental Disabilities, 41-42, 40-51. https:/ /doi.org/10.1016/j.ridd.2015.05.009

Hernando, I., Susana. (2014). El patinaje como actividad extraescolar dentro de la educación no formal: Desarrollo de la coordinación dinámica general y del equilibrio [Tesis de Maestría]. Universidad de Valldolid.

Hillis, T. L., \& Holman, S. (2014). The relatinship between speed and technical development in young speed skaters. International Journal of Sports Science \& Coaching, 9(2), 393-400.

Hodges, N. J., Hayes, S., Horn, R. R., \& Williams, A. M. (2005). Changes in coordination, control and outcome as a result of extended practice on a novel motor skill. Ergonomics, 48(1114), 1672-1685.

Hondt, E. D., Deforche, B., Gentier, I., De Bourdeaudhuij, I., Vaeyens, R., Philippaerts, R., \& Lenoir, M. (2013). A longitudinal analysis of gross motor coodination in overweight and obese children versus normal-weight peers. International Journal of Obesity, 37, 61-67.

Iivonen, S. (2016). A review of studies using the Körperkoordinationstest für Kinder (KTK). ResearchGate. https://www.researchgate.net/publication/ 301899260 A review of sudies using the Kapakoarinction for Kinder KTK

Kiphard, B. J., \& Schilling, F. (1974). Körperkoordinations Test für Kinder. Beltz Test GmbH, Weinheim.

Lech, G., Sertiæ, H., Sterkowicz, S., Sterkowicz Przybycieñ, K., Jaworski, J., \& Krawczyk, R. (2014). Effects of Different Aspects of Coordination on the Fighting Methods and Sport Skill Level in Cadet Judo Contestants. UĖINCI RAZLIÈITIH ASPEKATA KOORDINACIJE NA NAÈIN BORENJA I RAZINU SPORTSKE VJEŠTINE KADETSKIH JUDO NATJECATELJA., 46(1), 69-78.

Ledesma, R., Macbeth, G, \& Cortada de Kohan, N. (2008). Tamaño del efecto: Revisión teórica y apliaciones con el sistema estadístico. Revista Latinoamericana de Psicología, 40(3), 425-439.

Lloyd, R. S., \& Oliver, J. L. (2012). The Youth Physical Development Model: A New Approach to Long-Term Athletic Development. Strength and Conditioning Journal, 34(3), 61-72.https://doi.org/10.1519/SSC.0b013e31825760ea

Lopes, L. O., Lopes, V. P., Santos, R., \& Pereira, B. (2010). Associações entre actividade física, habilidades e coordenação motora em crianças portuguesas. Revista Brasileira de Cineantropometria e Desempenho Humano, 1521.https://doi.org/10.5007/1980-0037.2011v13n1p15

Lopes, V.P., Maia, J. A. R., Silva, R. G, Seabra, A., \& Morais, F. P. (2003). Estudo do nível de desenvolvimento da coordenação 
motora da população escolar (6 a 10 anos de idade) da Região Autónoma dos Açores. da região autónoma dos Açores. Revista Portuguesa de Ciências do Desporto, 3(1), 47-60.

López, D. C. J. (2014). El trabajo de coordinación en baloncesto. Análisis en categorías de formación. AGON International Journal of Sport Sciences, 4(1), 56-65.

Lyakh, V., Sadowski, J., \& Witkowski, Z. (2011). Development of Coordination Motor Abilities (CMA) in the System of LongTerm Preparation of Athletes. Polish Journal of Sport and Tourism, 18(3), 187-191.https://doi.org/10.2478/v10197-01100146

Martínez-González, M. Á., Sánchez-Villegas, A., Faulín, F. J., \& Toledo, A. E. A. (2014). Bioestadística amigable (Tercera). Elsevier.

Mateo, C. M., \& Sáez, M. S. C. (2004). Desarrollo físico y psicomotor en la etapa infantil. 27-64.

Maw, S., Proctor, L., Vredenburg, J., \& Ehlers, P. (2006). Influence of starting position on finishing position in World Cup $500 \mathrm{~m}$ short track speed skating. Journal of Sports Sciences, 24(12), 1239-1246.

Por la cual se establecen las normas científicas, técnicas y administrativas para la investigación en salud., Ministerio de Salud, 00843012 (1993).

Montenegro, O. A., Arjona., \& Ramos, S., Bermudez. (2015). La velocidad en el niño, Ritmo y Diferenciación. Kinesis.

Mori, H. R. (2008). La coordinación y motricidad asociada a la madurez mental en niños de 4 a 8 años. Universidad Femenina del Sagrado Corazón, 16(1), 139-154.

Muñoz, R., Daniel. (2009). La coordinación y el equilibrio en el área de Educación Física. Actividades para su desarrollo. www.efdeportes.com, 13(130). http://www.efdeportes.com/ efd130/la-coordinacion-y-el-equilibrio-en-el-area-deeducacion-fisica.htm

Pértegas, S., \& Pita, S. (2003). Cálculo del poder estadístico de un estudio Unidad de Epidemiología Clínica y Bioestadística. Complexo Hospitalario-Universitario Juan Canalejo. ACoruña (España) Actualización 20/01/2003. https:// www.fisterra.com/mbe/investiga/poder_estadistico/ poder_estadistico2.pdf

Petrone, N. (2006). Coordinación motora. https:// w $\quad$ w $\quad$ w

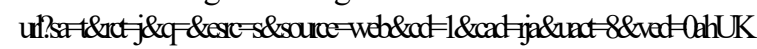
EvjmmtfuxqLMAhWEOyYKHaðMB88QFggaMAA\&ur hitp $\% / 3 \mathrm{~A} \% 2$ F\%2Fardilladigital.com\%2FDOCUMENTOS\%2FEDUCA CION\%2520ESPECIAL\%2FPSICOMOTRICIDAD\%2520$\% 2520$ FISIOTERAPIA $\% 2$ FCUALIDADES $\% 25$ 20MOTRICES\%2FCoordinacion\%2520Motora\%2520$\begin{array}{llllllllllllllllll}\% & 2 & 5 & 2 & 0 & \mathrm{P} & \mathrm{e} & \mathrm{t} & \mathrm{r} & \mathrm{o} & \mathrm{n} & \mathrm{e} & \% & 2 & 5 & 2 & 0 & -\end{array}$ \%2520art.pdf\&usg $=$ AFQjCNHUzPrTUiPlun0XdJhemwltSQY0g \&sig2=1vsPcildHgZyfQf3vfYlQg\&bvm=bv.119745492,d.dmo

Pita, S. (1996). Unidad de Epidemiología Clínica y Bioestadística. Complexo Hospitalario Juan Canalejo. A Coruña. Cad Aten Primaria. Actualización 06/03/2001., 3, 138-140.

Planelles, M. (2015). Mundial de patinaje de velocidad de 2015 en Kaohsiung (China Taipei) - 1rajornada - Los 300 m [Noticias]. Roller en linea. http://www.rollerenlinea.com/articles3858-mundial-de-patinaje-de-velocidad-de-2015-enkaohsiung-china-taipei-1ra-jornada-los-300-m.html

Quitério, A. L. D., Costa, J., Martins, M., Martins, J., Onofre, M., Gerlach, E., Scheuer, C., \& Herrmann, C. (2017). Educação Física: Avaliação das competências motoras em alunos de seis anos, do primeiro ano de escolaridade. Retos: nuevas tendencias en educación física, deporte y recreación, 31, 259-263.

Radwan, S. G (2014). The impact of development of the special coordination abilities on the general skill ability for table tennis juniors under 12 years old. International Journal of Science Culture and Sport (IntJSCS), 2(2), 30-42.

Rosero, R., Mónica Andrea, Palma, P., Luís Hebert, \& Dávila, A. A. (2012). Efecto de un programa de entrenamiento motriz sobre la agilidad y la coordinación en niño/as en edad escolar temprana de Tuluá. [Tesis de Maestría]. Universidad Autónoma de Manizales.

Ruiz, P. L. M. (2004). Competencia motriz, problemas de coordinación y deporte. Revista de Educación, 335, 21-33.

Sahan,A., Erman, K. A., \& Aðaoðlu, B. (2013). ANew Paradigm: Correlation between Laboratory and Field Tests of Coordination. Procedia - Social and Behavioral Sciences, 106, 164-169. https://doi.org/10.1016/j.sbspro.2013.12.019

Soto, Cegarra, Cuartero, López, \& Cantó. (2013). Desarrollo de las capacidades coordinativas a través del juego: Parkour. Revista Digital de Educación Física «Emásf», 4(20), 56-66.

Sparrow, W., \& Newell, K. (1994). The coordination and control of human creeping with increases in speed. Behavioural brain research, 63(2), 151-158.

Speedskating. (2016). FUNdamentals | Speed Skating Canada. http://www.speedskating.ca/about/skater-development/ fundamentals

Sterkowics, S., Lech, G., Jaworski, J., \& Ambrozy, T. (2012). Coordination motor abilities of judo contestants at different age. Journal of Combat Sports \& Martial Arts, 3(2), 5.

Stojanoviæ, N., Stojanoviæ, T., Stojanoviæ, D., Herodek, K., \& Jurko, D. (2014). The influence of coordination abilities on the precision of forearm passing in volleyball. DEFENDOLOGIJA, 1(35). https://doi.org/10.7251/ DEFEN1401003S

Tejero, C., Castro, M., \& Balsalobre, C. (2012). Importancia del tamaño del efecto. Una ejemplificación estadística con medidas de condición física / The importance of effect size: A statistical example using physical condition measurements. Revista Internacional de Medicina y Ciencias de la Actividad Física y el deporte, 12(48), 715-727.

Turvey, M. T. (1990). Coordination. American Psychologist, 45(8), 938-953. https://doi.org/10.1037/0003-066X.45.8.938

Van Waelvelde, H., De Weerdt, W., De Cock, P., \& SmitsEngelsman, B. C. M. (2004). Aspects of the validity of the Movement Assessment Battery for Children. Human Movement Science, 23(1), 49-60. https://doi.org/10.1016/ j.humov.2004.04.004

Vecino, J. D. (2011). El desarrollo y la evaluación de las capacidades coordinativas del balonmano. Propuesta metodológica para la iniciación deportiva. Secretaría de Educación de Veracruz. http://efisica.sev.gob.mx/difusion/ politicaeducinst20092010/pdfs/desarrollocapacbl.pdf.

Villa, C. R. (2011). Coordinación y equilibrio: Base para la educación física en primaria. Revista Digital: Innovación y Experiencias Educativas. N, 39, 1-11.

Walaszek, R., \& Nosal, T. (2014). Assessment of the Impact of One-Year Training in Acrobatic Rock'n'Roll on Overall Motor Coordination in Eight-Year-Old Children. Baltic Journal of Health and Physical Activity, 6(2). https://doi.org/10.2478/ bjha-2014-0009 